\title{
Orden republicano en Un Hombre, texto dramático del chileno Adolfo Urzúa Rosas*
}

\author{
Jorge Rueda Castro** \\ Orlando Aliaga Guzmán ${ }^{* * *}$
}

\section{Resumen}

En el artículo se analiza cómo el texto teatral Un Hombre (1913) de Adolfo Urzúa Rosas (1864-1937), representa una visión del mundo rural que aún después de 100 años del hecho independentista pone en escena algunas de las ideas y principios ideológicos a partir de los cuales se inauguró la república de Chile. Por lo anterior, se realiza un análisis crítico del mundo que muestra la obra, el que se codificaría en relaciones sociales de dominio expresadas a través de dicotomías sustentadas por la clásica dualidad civilización. Se concluye, por ende, que la creación de Urzúa conlleva un ideario con el fin de sumar al progreso civilizatorio y a la vida política del país por el que apostó el orden republicano hegemónico.

Palabras clave: ideología republicana, institucionalidad, contraposiciones semánticas.

\section{Republican order in a man, dramatic text of chilean Adolfo Urzúa Rosas}

\begin{abstract}
This article analyses how the dramatic text Un Hombre (1913), by Adolfo Urzúa Rosas (18641937), represents a vision of the rural world that even after 100 years of the Independence, sets on scene some of the ideas and ideological principles from which the Republic of Chile was founded. Base don the above, a critical analysis of the world shown by this work is carried out, analysis that would encode the social relationships of domain expressed by dicotomies supported by the classic duality civilization and barbarism. Finally, it is concluded that Urzua's creation entails an ideario with the end of adding to the civilization progress and to the political life of the country for which bet the republican hegemonic order.
\end{abstract}

Key words: republican ideology, institutionality, semantic contrapositions.

Las ideas desarrolladas en el presente estudio fueron expuestas en el XXVI Congreso Internacional de Teatro Iberoamericano y Argentino. Universidad de Buenos Aires, Facultad de Filosofía y Letras y Grupo de Estudios de Teatro Argentino y Latinoamericano (GETEA). 1 al 4 de agosto de 2017.

Chileno. Doctor en Estudios Americanos, mención Pensamiento y Cultura. Académico Universidad de Santiago de Chile. jorge.rueda@usach.cl

*** Chileno. Magíster en Literatura Latinoamericana y Chilena. Profesor Universidad de Santiago de Chile Santiago, Santiago, Chile. aliagaorlando80@gmail.com 


\section{Introducción}

La vida independiente alcanzada por Chile a principios del siglo XIX no provocó, como efecto posterior, la construcción de un estado-nación verdaderamente democrático. El hecho de que el proceso independentista se fundara sobre la herencia filosófica de la Ilustración, orientada a combatir la ignorancia de los hombres para conseguir su progreso y emancipación, provocó -paradojalmente- la imposición de valores universales que ignoró las visiones de mundo de territorios particulares. La elite decimonónica, y sobre todo la aristocracia criolla, que se sentía europea y pensaba como tal, poco o nada estableció en común con los intereses de los mundos mestizos. Además, aunque las ideas ilustradas llevaron a Camilo Henríquez (1769-1825) a declarar en 1812 desde las páginas de la Aurora de Chile que "la ilustración debe hacerse popular" (3), la clase dirigente (aristocracia criolla, que controló el proceso de independencia) profundizó en un sistema político-cultural de férrea dominación, inspirado ya por el mismo sistema colonial: "El distanciamiento entre ideología liberal y realidad social indica que [el país] vive todavía a medio camino entre el ancién régime y el mundo moderno, entre el Reino de Chile y la República Independiente" (Subercaseaux 22-23). La condición de 'europeos' nacidos en América los determinó a identificarse con los intereses dominantes; la elite criolla nacional, que ideológicamente se puso en contra del régimen hispano-colonial y buscó desespañolizarse, se bautizó a sí misma, paradojalmente, como 'aristocracia castellano-vasca' y aspiró, igualmente, a la acumulación de las riquezas que le proveía el monopolio del comercio local: "Fue el dinero del comercio el que permitió a la aristocracia castellano-vasca comprar al Rey de España sus mayorazgos y títulos de Castilla. O sea: su adherencia 'aristocrática'” (Salazar 13).

De este modo, la clase dirigente no apostó, al menos en los hechos, por un proyecto de República que incorporara la heterogeneidad social y cultural de lo colectivo para la construcción del naciente estado-nación. Esto último, incluso después de haber conseguido la independencia. Sus intereses estuvieron en el reclamo de un 'linaje' noble y europeo. Bajo esta perspectiva, se convirtió en una oligarquía dominante que conservó la visión dicotómica del 'yo' y el 'otro' al interior del proyecto republicano. Este ideario atravesó todo el siglo XIX y aún más. Un ejemplo de su persistencia, iniciado el siglo siguiente, se puede ver en la literatura que 
incluye en sí una concepción utilitaria a este proyecto: Un hombre (1913), obra dramática escrita por el chileno Adolfo Urzúa Rosas (1864-1937) y ambientada en la zona central de tipo rural.

En efecto, la organización laboral del mundo rural chileno se desarrolló bajo el despótico régimen de la hacienda. Una vez establecido el estado-nación, la hacienda no mejoró las condiciones de dominio y explotación de los peones u obreros agrícolas. Consecuencia de lo anterior, se entiende la progresiva deserción de los inquilinos de la hacienda, trabajadores que se convirtieron en peones-gañanes, es decir, temporeros que recorrieron el país y que, en algunos casos, cruzaron fronteras en franca fuga. Se originó, por este hecho social, el mito del roto, del buscavidas andariego, que buscó trabajo ocasional en medio, igualmente, de acciones condenadas por el orden institucional (como el robo, el saqueo y el asalto a la hacienda). Esto último, quizás, como una guerra en contra de los grupos dominantes. Conocido como un proceso social de 'peonización' o 'gañanería', se manifestó en Chile entre 1780 y 1940, y es descrito por los historiadores Pinto y Salazar (1999) en los siguientes términos:

Las masas populares [...] deambularon por décadas de una faena a otra [...]. En cada lugar, se hallaron forzadas a aceptar un mismo contrato laboral ("de peonaje"): salario desmonetizado (pago en "fichas"), trabajo forzado (arresto de vagabundos, enganche de presidiarios, deudas con las pulperías, etc.), castigos físicos (azote y cepo), servicio doméstico a mérito (sin salario), desprotección de la faena, revisiones abusivas, barracones infectos, monopolio comercial de las pulperías, etc. [...]. Por ello, los peones tendieron a escapar de las faenas, a asaltarlas y robarlas, desacatando a patrones, jueces, vigilantes y policías. Por décadas, la masa peonal actuó “alzándose en la faena” (o emigrando fuera del país), siguiendo un patrón conductual tal que podría decirse que el 'roto alzado' tenía su propio 'proyecto histórico' de resistencia: luchar contra la peonización (o escapar de ella). (146)

En el contexto anterior, y a modo de premisa, la obra dramática $U n$ hombre, escenificó 103 años después de la gesta independentista -con evidente intención ideológica y programática- la dicotomía clasificatoria del hombre y de la realidad en términos de 'civilización/barbarie', la cual, en el texto de Urzúa, promueve contraposiciones semánticas 
como aquélla del trabajador honrado que activa el progreso v/s el gañán o roto bandido, impulsor del desorden, del retraso civilizatorio y la violencia. Como se observa, las figuras del civilizado y del bárbaro (enfrentamiento base de la acción de Un hombre), alcanzaron en América Latina su formulación definitiva en la obra de Sarmiento. Se recordará que la antinomia expresaba, por un lado, las aspiraciones de la clase burguesa argentina en ascenso durante el siglo XIX, y más ampliamente latinoamericana; por el otro, la prevalencia de las ideas que buscaban la consolidación de un status favorable a los intereses de aquella clase social. Al interior de esas conceptualizaciones $U n$ hombre será considerado, en este estudio, como expresión del discurso hegemónico, el que tuvo como programa la confirmación del sistema de ideas de las elites seculares (promotoras del desarrollo del proyecto nacional y de la cultura oficial en Chile). En general, como se consigna en algunos estudios panorámicos del teatro en Chile, el texto se ajusta evidentemente al teatro costumbrista de comienzos del siglo XX (cf. Latorre, Rodríguez); también ha sido analizado como literatura libertaria anarquista (cf. Pereira), cuando la considera expresión de aquel tipo de teatro que "desarrolla un esquema de la acción en el cual la meta por alcanzar está mediatizada por un serie de dificultades que tienen que ser superadas, si se quiere satisfacer la pretensión de hacer realidad un nuevo orden de cosas" (167). Al respecto, este trabajo propone ampliar ambas concepciones al hacerlo dialogar con la ideología u orden republicano de vida que, en el imaginario cultural de la elite de Chile del centenario ${ }^{4}$, aún se preocupó por profundizar la dicotomía civilización (republicana)

\footnotetext{
4 Hacia 1913, año en que Urzúa obtuvo el segundo premio en el certamen del Consejo Superior de Letras con el texto dramático Un hombre, la idea de crisis social y política estuvo muy presente en sectores de la vida pública de Chile. Hacia 1910, al conmemorarse el centenario de vida independiente, intelectuales de diversas posiciones realizaban concienzudos análisis sobre la situación del país y denunciaban los males que la sociedad estaba generando. Para algunos, la 'Crisis del Centenario' tenía su origen en la decadencia moral de la clase política de la época. Para otros, sin embargo, la explicación debía buscarse en los conflictos sociales que se agudizaban día a día ante la mirada indiferente de la élite. Había pobreza en casi todas las ciudades del territorio. La indigencia y la mendicidad, hasta ese entonces cosas vagas, aparecían en las calles. Las condiciones de trabajo empeoraban y la brecha entre la riqueza y el lujo que ostentaba la clase dominante y la pobreza que se acentuaba en los sectores populares se ampliaba cada vez más. Fue evidente el problema moral al cual se vio enfrentada la clase política. La obra Un hombre, por ende, cobra fuerza al interior de este contexto al entenderla y proponerla en este estudio como un discurso de apoyo al proyecto oficial de la nación. Respecto al autor, es importante reconocer lo multifacético de sus actividades: de profesión odontólogo, fue además actor, profesor, director de teatro y de cine. Su filmación de 1910, Manuel Rodríguez, lo convierte en el primer director de una película argumental en Chile. Fundó la Compañía Dramática Nacional. También publicó algunas páginas sobre el arte de declamación. De su más destacado discípulo, el dramaturgo Antonio Acevedo Hernández (1886-1962), se conservan algunas notas referidas a Urzúa: “Autor de buenas comedias cimentadas sobre la base de la chilenidad más acendrada (...) Profundo crítico de sí mismo, gran conocedor de la
} 
/ barbarie (anti-institucionalidad). De este modo, la creación de Urzúa conlleva un ideario con el fin de sumar al progreso civilizatorio y de la vida política del país por el que apostó el orden republicano, es decir, "la instauración de una institucionalidad que garantice el orden público, entendiendo que éste descansa sobre el orden social" (Silva 21).

Dentro de aquel marco político-cultural, el texto como discurso social debió interpelar al aparataje institucional con la finalidad de reafirmar un proyecto de nación en que los mundos populares (adversidad social) no se reconocieron como parte integrante de este proyecto, a menos que respondieran y se ajustaran a éste sin inquietarlo. Por discurso social se comprende "todo aquello que se dice y se escribe en un estado de sociedad, todo lo que se habla y se representa [...]; los repertorios tópicos que en una sociedad dada organizan lo narrable y argumentable" (Angenot 17). Esta diversificación, como rasgo central de los discursos, liga o distingue a muchos de ellos de la práctica significante dominante. Con este último planteamiento, el texto dramático de Urzúa se piensa como expresión de los temas-repertorios que identificaron el programa oficial de la nación. De aquí que, tal como lo postula Marie Malcuzynski (1991), el texto debe entenderse: "No sólo como el producto de una práctica socio-ideológica [sino también] en interacción recíproca con las demás prácticas cognitivas" (21). De este modo, -por una parte- el autor de Un hombre se entenderá como sujeto enunciativo de "los discursos de su tiempo (que lo atraviesan y constituyen) y que se materializan en el texto" (Arán 74), y -por otra- de los postulados interpretativos de este artículo, que se conciben como el ejercicio "para aislar e interpretar los recortes semióticos de las prácticas sociales textualizadas” (Arán 74).

\section{Desarrollo}

En los hechos, la obra presenta como protagonistas a una pareja de jóvenes de un pueblo del campo chileno: Vicho y Mañunga. La historia muestra las circunstancias en las que se lleva adelante la relación de la pareja que tendría que terminar en matrimonio, dado que Vicho ha alcanzado su independencia económica al hacerse cargo de la administración de sus bienes agrícolas después de la muerte de su padre. Un bandido, como

técnica teatral. Sabía mucho de teatro (...) Fue maestro en el Conservatorio de Música y Declamación de Santiago" (40). 
tercer personaje protagónico (bandolero representante del fenómeno de marginalización como lo fue la peonización de la zona central), aparece en el pueblo como portador de violencia y misterio. Este personaje no respeta la vida pacífica del poblado e instala la amenaza como proyecto central de sus motivaciones. El punto culminante de Un hombre se da cuando el bandido se empeña en raptar a Mañunga, de quien se ha prendado, y la obliga a que huya con él. Ante esto, será el propio Vicho quien neutralizará la fuerza negativa restaurando el orden de la comunidad y de la pareja de jóvenes cuando se enfrente al bandido en un duelo con cuchillo, concretando así el encarcelamiento del gañán por parte de la policía que rastreaba sus pasos desde hacía tiempo.

El discurso espacializado de la acotación de apertura busca en Vicho la consolidación de un estatus favorable a los intereses del orden y la decencia: “A la izquierda, una pequeña ramada, debajo de la que hay el yunque de la herrería de Vicho y sus habitaciones; es decir, un rancho decente" (294). La idea de trabajador honrado, independiente, con reconocimiento social, se complementa en la misma escena con la prosperidad económica de Vicho, como dueño de las tierras donde vive. Así lo expresa Rafael, un campesino que comenta: “... ya vis que aemás de su oficio de herrero, que le da de sobra pa él y su maire, es dueño de too esto junto con los ranchos del lao y cinco cuadras de tierra" (295). Se trata, por ende, de perfilar a Vicho con la imagen de la prosperidad, la honradez y la visibilidad social. El tercer protagonista instala, así, el campo semántico del bandidaje, cargado por la sospecha, la desconfianza, la 'tirria' o rechazo social. De este modo, la normalidad del mundo agrícola se ve alterada como consecuencia de la acometida de este extraño que circula por el sector. Preocupa al conjunto de campesinos la actitud desafiante que manifiesta el hombre y la actitud opresiva que muestra respecto de la joven prometida de Vicho. Está marcado por los instintos y la violencia criminal. Este ámbito lo demuestra ya el primer diálogo entre dos campesinos, con que se abre la obra, en el que ni siquiera se aclara el verdadero nombre del personaje. Tan solo se le nomina como 'el aparecío':

RAFAEL. ¿Vos creís que ando siempre abiáo como el aparecío? CHAGO. ¿Tamién vos [...] le tenís tirria?

RAFAEL. ... Y te lo igo porque mientras te convidaba y tomabay con él, te preguntó una pila de cosas que no había pa qué 
preguntar, ni vos teníais pa qué contestar, ya vis. Y too eso con un desconocío.

CHAGO. No, desconocío no. La maire de Vicho, que lo divisó, dijo que no se equivocaba, que era el mesmo.

RAFAEL. ¿Cómo el mesmo?

CHAGO. Un sirviente de la Hacienda de la Punta, que le dio una puñalaíta a uno de los patroncitos porque lo pilló con su china. Claro que tuvo que echalas pa' que no lo agarraran; pues, que el caballerito casi se murió.

RAFAEL. ¡Y ahora se viene por aquí tan fresco...!

CHAGO. Como de eso hacen sus diez años, y como parece que no usa ná el mismo nombre de entonces, y como la peste que le dio después, según dicen, lo desfiguró algo.

RAFAEL. Güeno, pues no debe ser un hombre honrao el que se cambia de nombre y de apelativo. (294-295)

En efecto, y desde el inicio, el texto establece mediante las acotaciones y el diálogo de personajes la contraposición de fuerzas como andamiaje del mundo dramático; es decir, a partir del eje dual encarnado por Vicho y los valores que representa (trabajador decente, honrado) y 'el aparecío', un bandido que concentra los disvalores atribuidos desde el rumor social (bandido violento, asaltante, gañán intrigante). En el primer personaje se advierte, por ende, un discurso de formación doctrinaria que nutrirá creencias asentadas por los aparatos de la institucionalidad: el trabajador prudente, respetuoso de las leyes y costumbres sociales fijadas por el orden dominante; con el segundo, la precariedad del bandido y el descentramiento del sistema de ideas y valores del orden republicano. Matea, madre de Vicho, refuerza explícitamente tal binarismo: "Ese hombre que apareció tan chapiáo y gastando tanta plata, parece que quiere ser la mala sombra de mi Vicho" (296-297). La figura del bandido, protagonista que activa el conflicto central del texto de Urzúa, es la imagen de la anti-institución, el peón mestizo y del bajo pueblo, rebelde y pendenciero que no tiene cabida ni en el discurso ni en la realidad construida desde el cuño elite-céntrico. Se trata de un marginal. La intención que lleva el 'afuerino' es la de provocar una fricción en las relaciones de la pareja, con la finalidad de recoger los beneficios del posible quiebre sentimental de los jóvenes. 
Su plan comprende dos etapas al interior de la acción: el acoso amoroso y el conato de rapto.

La acción y el discurso dramáticos de Un hombre se hacen, en efecto, programáticamente dicotómicos. Acorde con la naturaleza del género literario a la cual pertenece el texto, ésta permite organizar sus núcleos temáticos en función de una construcción conceptual antitética, en la que la oposición de fuerzas en conflicto deja entrever un sistema de oposiciones de sentidos. Se muestra a continuación en qué consisten los juegos de antinomias que marcan la representación de realidad al interior del texto de Urzúa. La concepción dual del mundo, arroja una realidad escindida en dos sectores, cada uno con características que llevan a su estilización o a su condenación: civilización e institucionalidad republicana para el primer caso; bandidaje bárbaro y anti-institucional para el segundo. Por cierto, esta estructura se empalma directamente con el modelo impuesto desde la cultura de la elite, estigmatizando expresiones de la heterogeneidad cultural que pudiesen obstaculizar las creencias legitimadoras del orden oficial. En consecuencia, la contraposición semántica sobre la cual descansa la siguiente propuesta de lectura, apela a la polaridad 'orden institucional v/s caos anti-institucional'. Este binarismo encierra en la obra dos ejes o enclaves desde los que es posible codificar las contraposiciones: a) trabajador honrado / bandido asaltante; b) familia, en el sentido de pertenencia a las convenciones del orden / otras formas de convivencia excluidas del sistema de creencias oficiales.

Aquello que el conflicto dramático no revela es que 'el aparecío', junto con su cuadrilla de bandidos, es la consecuencia del proceso de peonización del bajo-pueblo. Urzúa tampoco hace mención a las condiciones de explotación y de trabajo forzado no-asalariado que imponía la hacienda. Entrega, sin embargo, la causa que explica, y en consecuencia denuncia, la conversión de 'el aparecío' en bandido: la apuñalada dada a uno de los hijos del patrón por abusar de su 'china'. Este hecho, recurrente al interior de la estructura de los terratenientes, fue parte del sistema de explotación que ejerció la hacienda. En el discurso oficial, sin embargo, al peón-gañán o roto alzado se le consideró revoltoso, pendenciero e inmoral. Fue el paradigma de la 'clase peligrosa'. En contraposición, Vicho es un artesano con taller, simbolización del esfuerzo familiar e individual que le ha asegurado un buen pasar económico, por lo que representa la clase de artesanos independientes con reconocimiento social. 
Las fuerzas entran en conflicto cuando 'el aparecío', que dice llamarse Mateo, se enamora de la Mañunga, la que responde con agrado en un primer momento, hecho que provoca los celos de Vicho. En este marco argumental, es posible leer el segundo eje de contraposiciones que ilumina el horizonte programático del texto: el trabajador honrado e independiente debe formar una familia, como lo promueve la convención institucional. En referencia al comportamiento ejemplar de Vicho, su madre le dirá a Mañunga: "Por vos está más sosegao y más trabajador que nunca!" (296). Y el propio Vicho expresará en función de este respecto: "Ende entonces comprendí que no poía apartarme de vos!, que no puéo vivir sin verte, sin sentir aentro e mí cuando te veo" (298). En las prácticas del bandidaje, en contraposición, el maridaje no pertenece necesariamente a sus códigos. El 'aparecío' no puede manejar la atracción impulsiva que experimenta por Mañunga: “... ésta me ha proucío a mí algo aquí aentro! Güeno, lo que es yo, no me voy en banda, aunque tuviera que encendele fuego a tóo el pueblo!” (306). Lo que el bandido expresa es la opción violenta de raptar a la mujer por la que siente atracción. Posiblemente, hacia los años iniciales del siglo XX aún perduraba en los campos de Chile algún sustrato de prácticas culturales originarias, donde el robo de la mujer fue parte del mundo convenido por algunos pueblos. Un ejemplo de esta dimensión está en el sentido de la costumbre ancestral de la organización mapuche, en que precisamente el weñezomon incluía el rapto de la mujer. En el contexto de divulgación y validación de las creencias oficiales, como lo hace el texto dramático en cuestión, esta dualidad demarca moral y culturalmente aquellas formas de convivencia no sancionadas por el orden institucional y que subyacen para entonces en el imaginario colectivo.

El atisbo de este segundo eje o enclave de contraposición refuerza, de paso, la enorme significación que implicó para el proyecto de nación el papel de la mujer en las dimensiones de la vida social y privada. El texto es más que explícito en atribuir como valores cruciales de la mujer: el recato, la prudencia y las convenciones sociales que destinaban a la mujer al hogar, en acuerdo con el relato masculino de la historia, ya en calidad de padre, ya en calidad de hermano o de marido. Y aunque en el caso de la obra comentada, se trata de la construcción social de una mujer joven y de campo, tales 'valores' pueden entenderse como aspectos de un proyecto social que trasciende lo particular y construye un sistema de valores más denso que se enfila hacia el contexto social. Mañunga, como personaje femenino principal, y como uno de los vértices del triángulo 
amoroso que teje la historia, se muestra rebelde y coqueta (como joven que es) en gran parte de la acción. No obstante, al interior de la premisa que guía esta lectura, su comportamiento sirve como modelo de lo que precisamente una mujer no debe hacer, de acuerdo con los postulados del republicanismo y la herencia española. Son varios los personajes que refuerzan estos principios. El campesino Rafael expresará estas ideas en los siguientes términos: “Claro se ve que ['el aparecío'] le anda buscando las tres mitás a la Mañunga, la hija e ño Pedro, que tóos saben que ha prometío casarse con Vicho y que ño Pedro es gustoso. Es la Mañunga que tiene esa maldita risa que parece que le da esperanza a tóo el mundo" (295). Matea, la madre de Vicho, será aún más dura en defender las convenciones de la 'decencia' que debe guardar la mujer comprometida con su hijo: "Me ijeron que ese que han dao en llamar el aparecío te está buscando las tres mitás, y que vos, ayer principalmente, estabay quiltreando con él. Dicen que vos le diste mucho lugar; que la cueca que bailaste con él fue muy apretá y que hasta le regalaste un clavel que él te pidió” (297). Y Vicho dirá: “¿Que qué me habís hecho? Y ayer..? Si viste que yo estaba enojao, ¿pa qué bailaste con él? Pa qué le regalaste el clavel? ¿Paqué te reíay?” (299).

Paralelamente, llegan noticias de que una cuadrilla de bandidos, liderada por un temido ladrón y famoso peleador de cuchillo, nominado como el Coronta, ha asaltado la Hacienda del Bajo, donde ha muerto el administrador y un sirviente. Todo esto despierta el miedo de los pobladores, ya que saben que la peligrosa banda ronda en las cercanías del pueblo:

RAFAEL. Güeno, y qué ha oído decir del salteo del bajo, ño Peiro?

PEDRO. Más u menos lo que Uds. saben al respeuto. Que el Coronta con su cuairilla se metió en casa del administrador del Bajo. Toos llevaban máscaras. Mataron al aministraor y a un sirviente y se llevaron más de trescientos pesos en plata y algunos efeutos de valor.

CHAGO. ¿Y los persiguieron?

PEDRO. Sí, pero parece que un poco tarde, porque los dejaron a toos amarraos [...] Yo creo que hace falta que aquí estemos toos prevenidos. En el pueblo somos muy pocos y apenas tuvimos armas. (300-301) 
En la parte final de la obra, cuando se inicia la resolución del conflicto dramático, se revela que Mateo o 'el aparecío’ es la misma persona que el Coronta, y que ha cambiado su identidad para entrar al pueblo y llevarse a la Mañunga. El enfrentamiento a cuchillo entre Vicho y 'el aparecío' cierra la acción del texto, la cual se resuelve, finalmente, en favor de Vicho, al dejar a Mañunga para el 'ganador". Pese a que el primero goza de fama de pendenciero y diestro en el uso del arma, ya que "manija el cuchillo como el cielo manija las estrellas" (245), es el Vicho quien triunfa en la pelea y le perdona la vida a su contendor, permitiéndole, además, la posibilidad de huir. No obstante lo anterior, el Coronta es prontamente apresado por la fuerza policial, que lo buscaba por el asalto y muerte del administrador de la Hacienda del Bajo. Este final deja en claro el sentido moralizador y programático de la obra. Por una parte, el peón-gañán o roto-bandido, quien quiso robar violentamente a la mujer comprometida, es derrotado, capturado por el orden policial ${ }^{5}$. Por otra, el Vicho, trabajador honrado y prudente, quien desea formar una familia acorde a las convenciones, triunfa. En consecuencia, los valores que el drama exalta son los del respeto al orden y a las leyes, a la propiedad privada y a la familia. El Coronta con su banda, en cambio, no sólo representan la reacción en contra del orden legal, en especial el del régimen hacendado, sino también en contra del orden familiar. El vivir sin destino fijo, de un lugar a otro, también es un factor que no constituye posibilidades para fundar familia, más aún si se escapa de la justicia.

\section{Conclusión}

En el marco de lo recién descrito, el interés que observa Urzúa por el mundo rural que describe en Un hombre, puede explicarse como respuesta del autor al clima social que observó hacia 1913 (tiempo del cen-

\footnotetext{
Hacia mediados del siglo XX en Chile, el discurso oficial del imaginario de la elite cultural dirigente continuaba creando ruindad en función del mundo popular y en especial de la imagen del roto. El intelectual Raúl Silva Castro escribió en función de este respecto: "Se dice de un hombre que comete rotadas, o roterías, cuando su comportamiento es sucio, cuando el individuo revela cobardía y mala intención, y si carece de franqueza y de concepto de la responsabilidad [...] La rotada, como sustantivo que denota a la multitud, señala sin dudas a la plebe, a la gente menos culta y más soez" ("No más roto chileno" 118). El mismo Silva Castro reconoce, en otro de sus escritos, la obligación que tiene la intelectualidad dominante de 'moldear' ideales públicos sobre la mayoría: "Los pueblos se moldean a compás de los ideales de sus clases dirigentes, que en ellos difunden por espontánea penetración las costumbres y los usos fomentados al calor de las formas más elevadas de la cultura. Esta penetración [...] se hace tanto más operante y vivaz cuanto más eficaces son los medios de que se sirven las clases superiores para influir sobre las otras" ("Verdejo y Anti-Verdejo" 105).
} 
tenario de la nación), generado por el peonaje en cuanto amenaza al orden republicano de Chile. La propuesta de lectura que este artículo ofrece del texto pasa por la valoración del hombre de campo que contribuye al orden capaz de construir la cultura nacional desde la perspectiva ideológico-dominante. El otro, el delincuente, el roto-gañán, representa el estereotipo del transgresor, del marginal y de lo extrasocial. Esta dualidad semántica encarnada en la pareja protagónica masculina se vuelve, por ende, un aspecto fundamental. La concepción de la literatura que exhibe el autor se inscribe, sin dudas, al interior del proyecto hegemónico que reconoce implícitamente que aún iniciado el siglo $\mathrm{XX}$ algunos sectores sociales no se sentían identificados por la patria ni experimentaban como propias sus instituciones. Esto concuerda con la versión que afirma que la imposición del Estado se realizó en Chile de tal manera que fueron extraídos sus principios democráticos. El proceso fue tan artificioso y forzado que, acorde con el historiador A. Jocelyn-Holt, entre 1830 y 1940 la oligarquía no tuvo otra salida que volcar todo cuanto estuviera a su alcance para crear una cultura nacional que le diera sentido: "Este esfuerzo extraordinario desde arriba resultó una 'comunidad imaginada' que se funda y que es, de hecho, la versión hegemónica del nacionalismo en la historia de Chile" (ctd. en Rojo 40). Obviamente, la creación desde arriba a la cual alude el historiador, resultó en la imposición de la comunidad imaginada desde el orden dominante, al interior del cual la literatura constituyó uno de sus códigos. La ausencia de verdaderas relaciones de igualdad fue el sello que la caracterizó.

De este modo, Urzúa procede a representar parte del mundo rural en el marco de los principios que abogaron por desterrar los vicios que amenazaban la ideología republicana. Esto, para fortalecer -acorde con la premisa que orientó este trabajo- el control de las instituciones aseguradoras del orden oficial. Los personajes de Un hombre muestran costumbres típicas, como apostar en carreras de caballo, bailar cueca y beber chicha. Muchos de los numerosos juegos y costumbres que practicó el bajo-pueblo, nunca fueron bien recibidos por la oligarquía debido a las continuas riñas que se producían por las apuestas y la bebida, pero sobre todo por los grupos que congregaban, lo que provocó el miedo de los aseguradores del orden institucional ante levantamientos o revueltas mayores.

El rescate que realiza Urzúa del mundo popular se centra en Vicho, trabajador que vive en concordancia con las aspiraciones del pueblo 
que hizo suyos los principios y valores de la honradez, la familia y la prudencia en cuanto categorías aseguradoras del respeto a las leyes y a la propiedad. Lo erige, por lo mismo, como el modelo masculino: un hombre capaz de saltar las crisis y contribuir en la construcción moral y cultural de la sociedad chilena de principios del siglo XX. En paralelo, el autor hace hincapié en una propuesta ideológica que refuerza la institucionalidad de la época, al denunciar como invirtuosas aquellas prácticas culturales impugnadas por la cultura oficial. En función de lo anterior, la obra pone en práctica una estética escénica en la que el espacio representado se ordena esquemáticamente respecto de las contraposiciones semánticas desarrolladas en el presente estudio. Ante la dualidad 'orden v/s caos', el texto establece la existencia de una 'violencia legítima', encarnada en Vicho cuando se enfrenta al 'aparecío' y una 'violencia ilegítima', encarnada precisamente en este último. La primera, restauradora del orden; la segunda, rupturista y desquiciadora del sistema institucionalizado.

\section{Referencias bibliográficas}

Acevedo Hernández, Antonio. Memorias de un autor teatral. Santiago de Chile, Nascimento, 1982.

Angenot, Marc. Interdiscursividades. De hegemonías y disidencias. Córdoba, Editorial Universidad Nacional de Córdoba, 1998.

Arán, Pampa Olga. "Migraciones del pensamiento de Bajtín. La sociocrítica en la perspectiva de M. Pierrette Malcuzynski”. Estudios, no. 17, Universidad Nacional de Córdoba - Centro de Estudios Avanzados (CEA), 2005, pp. 69-80.

Henríquez, Camilo. Aurora de Chile, jueves 7 de mayo de 1812, p. 3.

Latorre, Mariano. Memorias y otras confidencias. Santiago de Chile, Andrés Bello, 1971.

Malcuzynski, Marie Pierrette (Ed). “A modo de introducción”. Sociocríticas. Prácticas textuales. Cultura de fronteras. Ámsterdam, Atlanta, Rodopi, 1991, pp. 11-28.

Pereira, Sergio (Ed). Antología crítica de la Dramaturgia Anarquista en Chile. Santiago de Chile, Editorial Universidad de Santiago, 2005. 
Pinto, Julio y Gabriel Salazar. Historia contemporánea de Chile I. Estado, legitimidad, ciudadanía. Santiago de Chile, LOM, 1999.

Rodríguez, Orlando. Teatro Chileno (su dimensión social). Santiago de Chile, Quimantú, 1973.

Rojo, Grínor et. al. Postcolonialidad y nación. Santiago de Chile, LOM, 2003.

Salazar, Gabriel. Diego Portales. Monopolista, sedicioso, demoledor (Juicio ciudadano a un anti-demócrata). Santiago de Chile, Editorial Universidad de Santiago, 2010.

Silva Castro, Raúl. "No más roto chileno”. Estampas y ensayos. México, Fondo de Cultura Económica, 1968, pp. 117-120.

“Verdejo y Anti-Verdejo". Estampas y ensayos. México, Fondo de Cultura Económica, 1968, pp. 103-106.

Silva, Bárbara. Identidad y nación entre dos siglos. Patria Vieja, Centenario y Bicentenario. Santiago de Chile, LOM, 2008.

Subercaseaux, Bernardo. Historia de las ideas y de la cultura en Chile. Tomo I. Sociedad y cultura liberal en el siglo XIX: J. V. Lastarria. Santiago de Chile, Editorial Universitaria, 1997.

Urzúa, Adolfo. Un hombre. Drama en un acto y en prosa. Santiago de Chile, Imprenta de Meza Hnos. En Antología crítica de la Dramaturgia Anarquista en Chile. Ed. Sergio Pereira. Santiago de Chile, Editorial Universidad de Santiago, 2005, pp. 293-311. 